Check for updates

Cite this: RSC Adv., 2018, 8, 35139

Received 1st July 2018

Accepted 30th August 2018

DOI: $10.1039 / \mathrm{c} 8 \mathrm{ra05621h}$

rsc.li/rsc-advances

\section{The stability and bioaccessibility of fucoxanthin in spray-dried microcapsules based on various biopolymers}

\author{
Xiaowen Sun, ${ }^{a}$ Ying $\mathrm{Xu}{ }^{\mathrm{a}}$ Lili Zhao, $^{\mathrm{b}}$ Hongxue Yan, ${ }^{\mathrm{b}}$ Shuhui Wang ${ }^{\mathrm{c}}$ \\ and Dongfeng Wang $(\mathbb{D}$ *a
}

Fucoxanthin is a major marine carotenoid with many biological activities. It is well known that fucoxanthin is unstable to heat and acid due to its polyunsaturated structure. Another defect of fucoxanthin is the low bioavailability and all these drawbacks make it limited in the food industry. In order to improve its stability and intestinal absorption, fucoxanthin was encapsulated with biopolymers by spray drying in this study. All the microspheres we prepared had a spherical shape with encapsulation efficiencies (EE) ranging from $86.48 \%$ to $97.06 \%$. A heat stability test showed that maltodextrin (MD), gum arabic (GA) and whey protein isolate (WPI) improved the thermal stability of fucoxanthin. The degradation kinetics of the loaded fucoxanthin encapsulated with biopolymers also indicated that GA and MD had a better protective effect on fucoxanthin. The encapsulated fucoxanthin degradation rate within MD, GA, WPI and gelatin (GEL) followed second-order kinetics and their half-life $\left(t_{1 / 2}\right)$ was $92.6 \mathrm{~d}, 99.8 \mathrm{~d}, 50.9 \mathrm{~d}$ and $69.3 \mathrm{~d}$, respectively. The simulated digestion test in vitro suggested that MD, GA and WPI effectively protected fucoxanthin in the gastric acid environment, meanwhile increasing the release rate of fucoxanthin in the intestinal tract.

\section{Introduction}

As the most abundant carotenoid in nature, fucoxanthin is found in edible brown algae such as Undaria pinnatifida, Sargassum fusiforme and Laminaria Japonica. In recent years, fucoxanthin has gained great research interest for its numerous beneficial properties, such as anti-obesity, ${ }^{1,2}$ anti-diabetic, ${ }^{3}$ antioxidant, ${ }^{4}$ anti-inflammatory ${ }^{5,6}$ and anti-cancer ${ }^{7,8}$ activities. Considering its excellent bioactivities and pharmacological effects, fucoxanthin can potentially be developed into a nutritional supplement or as a promising drug for human health.

Fucoxanthin, with a distinct structure including an unusual allenic bond, epoxide group, and conjugated carbonyl group in polyene chain, is susceptible to external conditions. Oxygen, light, high temperature, heavy metals exposure during the processing and storage can accelerate the degradation of fucoxanthin, ${ }^{9-11}$ and hence restrict its utilization. Therefore, stability is an important factor when fucoxanthin is used as the colorant and bioactive ingredient in food and pharmaceutical industries. Furthermore, Hashimoto et al. ${ }^{12}$ reported that the

${ }^{a}$ College of Food Science and Engineering, Ocean University of China, Qingdao 266003, People's Republic of China. E-mail: wangdf@ouc.edu.cn

${ }^{b}$ State Key Laboratory of Bioactive Seaweed Substances, Qingdao Brightmoon Seaweed Group Co Ltd, Qingdao, 266400, People's Republic of China

'Qingdao Municipal Center for Disease Control \& Prevention, Qingdao 266033, People's Republic of China bioavailability of fucoxanthin was relatively low. Therefore, the weak stability and limited bioavailability are the major factors restricting the wider application of fucoxanthin.

Microencapsulation is an effective approach to prevent rapid degradation of sensitive compounds and increase the bioaccessibility of them. ${ }^{13,14}$ Spray-drying is a common technique to prepare microcapsules with a relatively low water content and water activity to reduce the risk of physicochemical degradations..$^{15}$ In addition, the selection of proper wall materials is crucial for the effective microencapsulation of bioactive compounds. The wall materials can protect the sensitive ingredients against some adverse reactions and control the release of them. Proteins (whey proteins, gelatin), oligosaccharides (maltodextrins, cyclodextrin), and polysaccharides (GA, alginates) are commonly used as encapsulation materials. GA, as one of the most common wall materials used in microencapsulation by spray drying, is a good wall material encapsulating carotenoid, such as $\beta$-carotene, ${ }^{16}$ lycopene, ${ }^{17}$ astaxanthin. ${ }^{18,19}$ Maltodextrin is a starch produced by partial acidolysis or enzymolysis. It has a low cost, viscosity and a good pigment protection against oxidation. ${ }^{20,21}$ Hydroxypropyl- $\beta$ cyclodextrin (HP- $\beta$-CD), a hydroxyalkyl derivative of $\beta$-cyclodextrin, could enhance the storage stability of astaxanthin/ canthaxanthin by inclusion of astaxanthin/canthaxanthin in HP- $\beta-C D .^{22,23}$ Recent studies also indicated that whey proteins, gelatin or pea protein could deliver various dietary carotenoids, such as $\alpha$-carotene,,$^{24}$ astaxanthin, ${ }^{25}$ and lutein. ${ }^{26,27}$ 
The object of this study was to select proper wall materials to encapsulate fucoxanthin and improve its stability and bioaccessibility effectively. The characteristics of the fucoxanthin microcapsules prepared by different wall materials were investigated. In addition, the thermal stability of the fucoxanthin microcapsules while the degradation kinetics of diverse fucoxanthin microspheres was studied. Furthermore, the digestive characteristics were evaluated in simulated digestion process.

\section{Materials and methods}

\subsection{Materials}

Dried Undaria pinnatifida was supplied by Jiayi Aquatic Products Co. Ltd. (Shandong, China) and was pulverized and sieved (200 mesh sieve). HP- $\beta$-CD was purchased from Zhiyuan BioTechnology Co. Ltd. (Shongdong, China). MD (DE = 15-20) was purchased from Xiwang Sugar Co. Ltd. (Shandong, China). GA was purchased from Solarbio Science \& Technology Co. Ltd (Beijing, China). Isolated pea protein (PPI) was purchased from Sciphar Natural Products Co., Ltd (Shaanxi, China). WPI was purchased from Hilmar Ingredients (California, USA). HPLCgrade acetonitrile, methyl tertiary butyl ether (MTBE), fucoxanthin standard, and gelatin were purchased from Sigma Chemical Co. (St. Louis, MO). All other reagents used were of analytical grade.

\subsection{Preparation of fucoxanthin}

Fucoxanthin was prepared as described in our previous paper, ${ }^{28}$ briefly, $1 \mathrm{~kg}$ dry powder of Undaria pinnatifida was extracted thrice in $4 \mathrm{~L}$ of $80 \%$ ethanol at $40{ }^{\circ} \mathrm{C}$ for $12 \mathrm{~h}$, including $0.5 \mathrm{~h}$ for stirring process and $11.5 \mathrm{~h}$ for static extraction. The extract was filtered and condensed under reduced pressure, and then treated with $95 \%(\mathrm{v} / \mathrm{v})$ methanol $/ n$-hexane $(1: 1, \mathrm{v} / \mathrm{v})$ to isolate fucoxanthin. The methanol layer containing fucoxanthin was concentrated and subjected to silica-gel adsorption chromatography. Fractions with different colors were eluted with petroleum ether/ethyl acetate (the volume ratio was reduced from $7: 3$ to $1: 9)$ successively, the reddish-orange ones containing fucoxanthin were condensed in vacuo at $37^{\circ} \mathrm{C}$ and dried under nitrogen. Then the residue was dissolved in $30 \mathrm{~mL}$ acetonitrile and stored at $-20{ }^{\circ} \mathrm{C}$ until use. The whole process should avoid light as possible.

\subsection{Quantitative analysis}

To quantify fucoxanthin prepared, a sample from the reddishorange fractions was diluted with acetonitrile and filtered with a $0.22 \mu \mathrm{m}$ polytetrafluoroethylene membrane filter for analysis by an HPLC system (1200, Agilent Technologies Inc., Santa Clara, CA, USA). Detection procedure consulted the method described by Rivera and Canela-Garayoa ${ }^{29}$ with some modifications: a ZORBAX Eclipse XDB-C18 column $(4.6 \times 250$ mm, $5 \mu \mathrm{m}$, Agilent Technologies Inc., Palo Alto, CA, USA) was used for fucoxanthin separation using a tertiary solvent gradient elution: initial solvent composition consisted of $80 \%$ acetonitrile, $20 \%$ water $(\mathrm{v} / \mathrm{v})$, changed to $72 \%$ acetonitrile, $18 \%$ water, and $10 \% \mathrm{MTBE}$ in $10 \mathrm{~min}$ and persisted for $10 \mathrm{~min}$.
Initial conditions were reestablished in $5 \mathrm{~min}$, and the column was re-equilibrated for $7 \mathrm{~min}$ before the next injection. The flow rate was $0.8 \mathrm{~mL} \mathrm{~min}{ }^{-1}$ with a column temperature of $25^{\circ} \mathrm{C}$, and $10 \mu \mathrm{L}$ samples were injected into the column. Fucoxanthin was detected at the wavelength of $450 \mathrm{~nm}$ and its concentration was determined according to the standard curve obtained on the same conditions.

\subsection{Preparation of $\mathrm{o} / \mathrm{w}$ emulsion}

The stock solutions of coating materials were prepared by mixing $40 \mathrm{~g}$ of polysaccharide with $150 \mathrm{~mL}$ of distilled water using magnetic stirrer (RCT basic, IKA, Staufen, Germany). In the case of protein, their solutions were prepared by dispersing $20 \mathrm{~g}$ of protein powder into $150 \mathrm{~mL}$ of distilled water, mixing continued until the materials were dissolved well, the $\mathrm{pH}$ of the protein solutions were adjusted to 7.0 using $1 \mathrm{M} \mathrm{HCl}$ and $1 \mathrm{M}$ $\mathrm{NaOH}$ solution if required, and then stand overnight at $4{ }^{\circ} \mathrm{C}$. The ethanol was driven out and the fucoxanthin was dispersed in coin oil. A coarse emulsion was prepared by dispersing fucoxanthin oil into $50 \mathrm{~mL}$ of a $1 \mathrm{mM}$ Tween 80 aqueous solution and the final concentration of fucoxanthin was $0.25 \mathrm{mM}$. The pre-emulsion was prepared by mixing the stock solution with the coarse emulsion respectively and homogenized intensively (10 $000 \mathrm{rpm}, 5 \mathrm{~min}$ ) using a high-speed blender (IKA T25 Digital Ultra-Turrax, Staufen, Germany). The emulsion was further homogenised by ultrasonic emulsification process (SCIENTZ-IID, Ningbo, China) for $10 \mathrm{~min}(500 \mathrm{~W}$, with a duty ratio of $66.67 \%$ ) and gentle magnetic stirring for $2 \mathrm{~h}$.

\subsection{Spray-drying of $\mathrm{o} / \mathrm{w}$ emulsion}

The prepared emulsion was dried using a spray dryer (LPG-5, Jiangsu, China) with rotary atomization at $170 / 90{ }^{\circ} \mathrm{C}$ inlet/ outlet temperature. After spray drying process, uniform powder was received and collected in plastic automatic sealing bags for the following study, respectively.

\subsection{Characterization of the microcapsule}

2.6.1 Microcapsules morphology and particle size distribution. Scanning electron microscope (SEM) was used to investigate the surface morphology and the microstructural properties of the six samples. The encapsulated powders were spread on circular aluminium stubs with a double-sided sticky tape, coated with a very thin layer of gold and examined using a SEM (JSM-840 A, Jeol, Ltd., Tokyo, Japan) at an accelerating voltage of $15 \mathrm{kV}$. Images were taken with magnification of 1000 times.

To obtain the average size and size distribution, the diameters of at least 300 particles were measured for each formulation using Image-Pro Plus 6.0 software (Media Cybernetics, Silver Spring, USA).

2.6.2 Moisture content. The determination of water content in the sample was carried out by drying in a vacuum oven at $60{ }^{\circ} \mathrm{C}$ until constant weight.

2.6.3 Water activity $\left(\boldsymbol{a}_{\mathrm{w}}\right)$. An Aqua Lab water 4 TE activity meter (Decagon Devices, Inc., WA, USA) was used to measure $a_{\mathrm{w}}$ 
of the encapsulated powders at ambient temperature $\left(25 \pm 0.1^{\circ} \mathrm{C}\right)$.

2.6.4 Encapsulation efficiency (EE) analysis. The EE was determined using the methodology described by Wang et al. ${ }^{30}$ with some modifications. In order to determine the amount of surface fucoxanthin, $0.5 \mathrm{~g}$ of dried microcapsule powder was dispersed in $20 \mathrm{~mL} n$-hexane and vortexed for $30 \mathrm{~s}$. The organic phase containing fucoxanthin was removed and the powder residue was washed with hexane $(2.5 \mathrm{~mL})$ twice. The solvent was evaporated under nitrogen stream and the residue was redissolved in $10 \mathrm{~mL}$ acetonitrile before HPLC analysis. Another $0.5 \mathrm{~g}$ of powder was dissolved in $20 \mathrm{~mL}$ water and segregated with a high-speed disperser at $10000 \mathrm{rpm}$ for $60 \mathrm{~s}$. In order to quantify the total amount of fucoxanthin in the encapsulated powder, the supernatant was collected after centrifugation and the extract process was repeated thrice. EE was calculated according to eqn (1).

$$
\mathrm{EE}(\%)=\frac{\mathrm{TF}-\mathrm{SF}}{\mathrm{TF}} \times 100
$$

where TF is the total amount of fucoxanthin present in the encapsulated powder, $\mu \mathrm{g}$; SF is the amount of fucoxanthin on the surface of the microcapsules, $\mu \mathrm{g}$.

2.6.5 Structure characterization. Fourier transform infrared (FTIR) was used to study the structural characterization of the six microcapsule samples. The FTIR spectra of fucoxanthin, HP- $\beta$-CD, MD, GA, PPI, WPI, GEL, Fx-HP- $\beta$-CD, Fx-MD, FxGA, Fx-PPI, Fx-WPI and Fx-GEL were obtained using a Nicolet iS10 spectrophotometer (Thermo Fisher Scientific, Waltham, MA, USA) in transmission mode. All of the samples were mixed with $\mathrm{KBr}$ at a ratio about $1: 100(\mathrm{w} / \mathrm{w})$. All the spectra were obtained from the average of 40 scans with a resolution of $4 \mathrm{~cm}^{-1}$ over a continuous spectral range of $400-4000 \mathrm{~cm}^{-1}$.

\subsection{Heat stability of fucoxanthin in microcapsules}

The samples based on different wall materials were placed in sealed tubes and covered with aluminum foil, which were then stored at $90^{\circ} \mathrm{C}$ for heat stability studies. One hundred milligram of each sample was taken each time and the pigment content was evaluated using HPLC.

\subsection{Kinetics modelling of fucoxanthin degradation in microcapsules}

Fucoxanthin microcapsules with different wall materials were placed in sealed tubes and covered with aluminum foil. The samples were then stored at $37{ }^{\circ} \mathrm{C}$ to study the degradation model. One hundred milligram of each sample was taken out weekly and the content of fucoxanthin was detected by HPLC. Fucoxanthin degradation was simulated with the reaction rate.

The kinetic model of the fucoxanthin degradation profiles was performed using Origin Pro 8.0 software (OriginLab, Northampton, MA, USA) to fit zero-order (eqn (2)), first-order (eqn (3)) or second order kinetics (eqn (4)) and the half-life $\left(t_{1 / 2}\right)$ was calculate by eqn (5)-(7).

$$
C-C_{0}=-k t
$$

$$
\begin{gathered}
\ln C-\ln C_{0}=-k t \\
1 / C-1 / C_{0}=-k t \\
t_{1 / 2}=C_{0} /(2 k) \\
t_{1 / 2}=\ln 2 / k \\
t_{1 / 2}=1 /\left(k C_{0}\right)
\end{gathered}
$$

where $C_{0}$ is the initial concentration of fucoxanthin, $\mu \mathrm{g} \mathrm{g}^{-1}$ powder; $C$ is the fucoxanthin concentration at time $t, \mu \mathrm{g} \mathrm{g}^{-1}$ powder; $k$ is the reaction rate constant, and $t$ is the storage time, d.

\subsection{In vitro simulated digestion}

The simulated digestion in vitro of microencapsulated fucoxanthin was conducted referred to a recent literature with some modifications. ${ }^{31}$ The following simulated digestive steps were performed in sequence. Briefly, $0.4 \mathrm{~g}$ dried microcapsule powder was dispersed in $14 \mathrm{~mL}$ of salt solution $(120 \mathrm{mM} \mathrm{NaCl}$, $5 \mathrm{mM} \mathrm{KCl}$, and $6 \mathrm{mM} \mathrm{CaCl}_{2}, \mathrm{pH} 2.2$ ) and then $1 \mathrm{~mL}$ of porcine pepsin $\left(75 \mathrm{mg} \mathrm{mL}^{-1}\right.$ in $0.1 \mathrm{M} \mathrm{HCl}$ ) was added. The suspension was incubated at $37^{\circ} \mathrm{C}$ shaking at $95 \mathrm{rpm}$ for $2 \mathrm{~h}$. In order to simulate the actual situation, the intestinal segments were divided into duodenum, jejunum, and ileum three sections. Primarily, $1 \mathrm{~mL}$ of enzyme mixture containing pancreatic lipase (5 $\mathrm{mg} \mathrm{mL}^{-1}$ ), pancreatin (40 $\mathrm{mg} \mathrm{mL}^{-1}$ ), and bile extract $(250 \mathrm{mg}$ $\mathrm{mL}^{-1}$ ) was added and the $\mathrm{pH}$ was raised to 5.5 by adding $0.9 \mathrm{M}$ sodium bicarbonate. The suspension was incubated for $30 \mathrm{~min}$ at $37^{\circ} \mathrm{C}$ to simulate the duodenum stage. And then the $\mathrm{pH}$ was adjusted to 6.0 and the samples were incubated for $90 \mathrm{~min}$ at $37{ }^{\circ} \mathrm{C}$ to mimic the jejunum stage. Finally, the $\mathrm{pH}$ of each sample was increased to 7.0 and incubated for $5 \mathrm{~h}$ at $37^{\circ} \mathrm{C}$ to complete the ileum phase of the digestion process. At the end of each stage, adequate volume of digestive juice was taken out and fucoxanthin was extracted with the mixture of acetone and $n$-hexane $(1: 1, \mathrm{v} / \mathrm{v})$. The retention rate of fucoxanthin in stomach and the release rate of fucoxanthin in intestine were detected by HPLC and calculated by eqn (8) or (9)

$$
\begin{aligned}
& R_{\mathrm{et}}(\%)=F_{1} / F_{0} \times 100 \\
& R_{\mathrm{el}}(\%)=F_{2} / F_{0} \times 100
\end{aligned}
$$

where, $F_{1}$ is the retained amount of fucoxanthin in the simulated gastric media, $\mu \mathrm{g} ; F_{2}$ is the released amount of fucoxanthin in the simulated intestinal media, $\mu g ; F_{0}$ is the total amount of fucoxanthin loaded in microcapsules initially added, $\mu$.

\subsection{Statistical analysis}

All experiments were performed on triplicate samples in replicates and the data were expressed as the mean \pm SD. Statistical analysis was performed using one-way analysis of variance (ANOVA) and Duncan's multiple comparisons in SPSS (version 
19.0, SPSS Inc., Chicago, IL, USA). The statistical significance was established when $p<0.05$.

\section{Results and discussion}

\subsection{Preparation of fucoxanthin from Undaria pinnatifida}

The fucoxanthin used in this study was initially isolated from Undaria pinnatifida by conventional solvent extraction, and further purified by silica gel column chromatography. The purified fucoxanthin had a relative content of up to $93.29 \%$ detected with HPLC according to method recommended in 2.3.

\subsection{Surface morphology of fucoxanthin loaded microcapsules}

The fucoxanthin microcapsules using HP- $\beta-C D$, MD, GA, WPI, PPI and GEL as wall materials were prepared by spray drying. The morphological characterization of the six kinds microspheres was observed using SEM, and the major morphology could be observed in Fig. 1. In general, the microspheres had a spherical shape with a rough surface. As shown in Fig. 1A-C, the microspheres prepared with carbohydrate polymers possessed a higher proportion of spherical shape with a smooth surface. As for protein wall materials (Fig. 1D-F), the particles appeared a rougher and more concave surface than those prepared with carbohydrate polymers. Probably because carbohydrate polymers could provide greater elasticity and good film forming property during the drying process.

\subsection{Size distribution of fucoxanthin loaded microcapsules}

The microcapsules size distribution was determined with the aid of the image analysis system (Image Pro-plus® 6.0). In order to compare the size distribution of the six microparticles, the average particle diameters and standard deviation were measured, and the results are shown in Table 1 . It can be observed that the mean particle diameter of the protein based microparticles $(8.67-9.26 \mu \mathrm{m})$ tended to be a bit bigger than the carbohydrate based ones $(6.55-7.18 \mu \mathrm{m})$.

\subsection{Moisture content and water activity}

Moisture content is one of the factors which could affect the shelf life of dried products. The moisture content of microcapsules is shown in Table 1 with an average value ranged from $2.68 \%$ to $4.90 \%$ (dry base). MD microcapsules had the lowest moisture content, which was similar to or lower than the values in other reports. For example, the $\beta$-carotene microcapsules
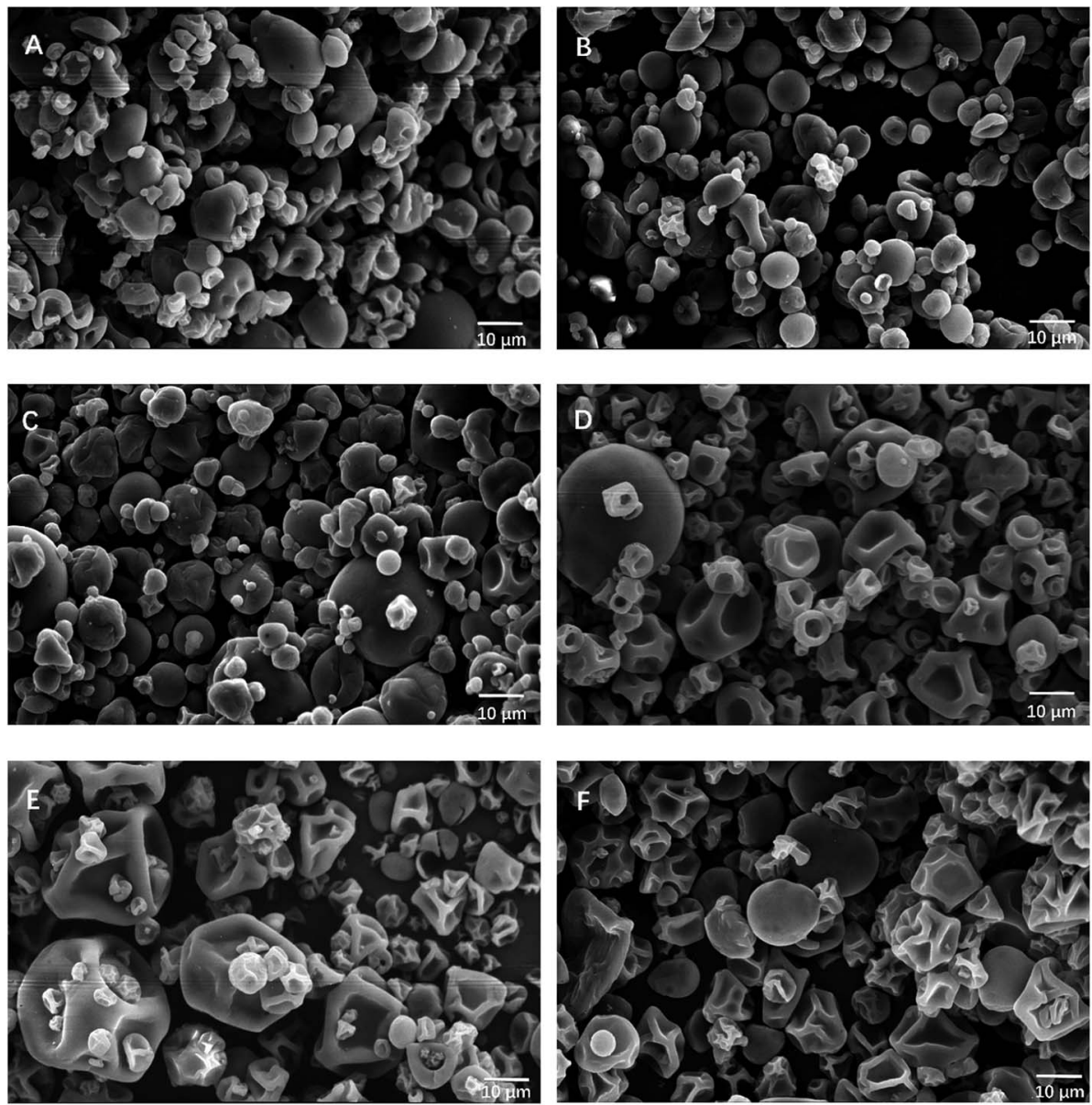

Fig. 1 SEM photographs of the microspheres containing fucoxanthin using different wall materials (A) HP- $\beta-C D$, (B) MD, (C) GA, (D) WPI, (E) PPI, and (F) GEL. 
Table 1 Characterization of fucoxanthin microcapsules with different wall materials ${ }^{a}$

\begin{tabular}{lllll}
\hline & Size $(\mu \mathrm{m})$ & Moisture content $(\%)$ & $a_{\mathrm{w}}$ & EE $(\%)$ \\
\hline HP- $\beta$-CD & $6.98 \pm 0.41^{\mathrm{b}}$ & $3.26 \pm 0.11^{\mathrm{b}}$ & $0.15 \pm 0.01^{\mathrm{c}}$ & $9.14 \pm 0.01^{\mathrm{c}}$ \\
MD & $6.55 \pm 0.10^{\mathrm{c}}$ & $2.68 \pm 0.04^{\mathrm{c}}$ & $0.14 \pm 0.36^{\mathrm{a}}$ \\
GA & $7.18 \pm 0.08^{\mathrm{b}}$ & $3.60 \pm 0.19^{\mathrm{b}}$ & $0.01^{\mathrm{c}}$ & $92.35 \pm 0.44^{\mathrm{b}}$ \\
WPI & $8.67 \pm 0.24^{\mathrm{a}}$ & $4.90 \pm 0.17^{\mathrm{a}}$ & $0.25 \pm 0.01^{\mathrm{a}}$ & $86.48 \pm 0.71^{\mathrm{e}}$ \\
PPI & $8.87 \pm 0.19^{\mathrm{a}}$ & $3.74 \pm 0.11^{\mathrm{b}}$ & $0.23 \pm 0.02^{\mathrm{ab}}$ & $90.88 \pm 0.14^{\mathrm{c}}$ \\
GEL & $9.26 \pm 0.35^{\mathrm{a}}$ & $3.47 \pm 0.19^{\mathrm{b}}$ & $0.21 \pm 0.01^{\mathrm{b}}$ & $90.08 \pm 0.10^{\mathrm{d}}$
\end{tabular}

${ }^{a}$ The different letters represent significant difference $(p<0.05)$.

prepared by spray drying using MD as wall material had a moisture content of $3.5 \% .^{32}$ Comunian et $a l .{ }^{33}$ reported moisture content was around $10 \%$ for spray-dried chlorophyllide microcapsules using GA, MD and soy protein isolatebased carrier systems. It has been reported that when moisture content attained values lower than $7 \%$, water diffusion through the food matrix decreases, which reduces the effect of moisture content on the physicochemical properties of the microcapsules and the accessibility of oxygen to pass the vesicular structure. ${ }^{34}$ Therefore, the microspheres we prepared should have a good stability during shelf life.

All the microcapsules showed low $a_{\mathrm{w}}$ values, ranging from 0.14 to 0.25 (Table 1). All of these $a_{\mathrm{w}}$ were adequate for the microsphere powders since low $a_{\mathrm{w}}$ could prohibit microbial growth and decrease degradation of components in the powders. ${ }^{34,35}$ In this study, the low moisture content and $a_{\mathrm{w}}$ caused a decrease in the relative rate of oxidation reactions, and consequently stabilized the fucoxanthin encapsulated in the microspheres.

\subsection{EE of fucoxanthin microcapsules}

In order to evaluate different wall materials' ability for fucoxanthin encapsulation, total and free fucoxanthin of the powders were determined and EE was calculated. As shown in Section 2.6, free fucoxanthin was the part extracted with organic solvents, and then EE values could reflect not only the rate of the fucoxanthin embedded in the particles but also the degree that the wall materials can prevent the leakage of encapsulated fucoxanthin. ${ }^{35}$ The detail data are presented in Table 1. The EE of the six microsphere powders ranged from $86.48 \%$ to $97.06 \%$. The EE values in this study were a little better than or similar to earlier reports. ${ }^{31,36,37}$ These results indicated the use of all these materials may yield a good encapsulation efficiency for carotenoid.

\subsection{Structure analysis of fucoxanthin microcapsules by FTIR}

The chemical binding status between fucoxanthin and six wall materials were studied by FTIR. Fig. 2 shows FTIR spectra in the wavelength range of $400-4000 \mathrm{~cm}^{-1}$. Fig. $2 \mathrm{~A}$ showed bands specific to fucoxanthin: the peak at $3419 \mathrm{~cm}^{-1}$ was assigned as O-H stretching. $3000-2800 \mathrm{~cm}^{-1}$ (C-H stretching), and 1339$1452 \mathrm{~cm}^{-1}$ (C-H scissoring and bending), typical for carotenoids, $1732 \mathrm{~cm}^{-1}(\mathrm{C}=\mathrm{O}$ stretching $)$ and $1032 \mathrm{~cm}^{-1}(-\mathrm{C}-\mathrm{O}$ stretching), typical for esters. The peak at $1928 \mathrm{~cm}^{-1}$ was assigned as an allenic bond, ${ }^{31}$ which is considered as a unique functional group of fucoxanthin. The peak at $968 \mathrm{~cm}^{-1}$ was for absorption band of $\mathrm{C}-\mathrm{H}$ in $\mathrm{C}, \mathrm{C}$ conjugate system and $1657 \mathrm{~cm}^{-1}$ was for $\mathrm{C}=\mathrm{C}$ stretching vibration.

Fig. $2 \mathrm{~B}-\mathrm{G}$ shows the FTIR spectra of $\mathrm{HP}-\beta-\mathrm{CD} / \mathrm{MD} / \mathrm{GA}$ and fucoxanthin loaded HP- $\beta-\mathrm{CD} / \mathrm{MD} / \mathrm{GA}$. In the IR spectrum of HP$\beta$-CD (B) and MD (D), there were many similar bands, $3385 \mathrm{~cm}^{-1}$ (O-H stretching), 2929 or $2926 \mathrm{~cm}^{-1}$ (C-H stretching), $1647 \mathrm{~cm}^{-1}\left(\mathrm{C}=\mathrm{O}\right.$ stretching), 1459 and $1409 \mathrm{~cm}^{-1}(\mathrm{C}-\mathrm{H}$ bending), $1373 \mathrm{~cm}^{-1}$ (O-H bending), $1155 \mathrm{~cm}^{-1}$ (C-O stretching). The infrared spectrum of gum arabic (F) possessed typical bands in the regions of $600-3000 \mathrm{~cm}^{-1}$. Some characteristics of absorption spectrum of GA were $3423 \mathrm{~cm}^{-1}$ (O-H stretching), $2930 \mathrm{~cm}^{-1}$ (C-H stretching), $1609 \mathrm{~cm}^{-1}$ (N-H bending), $1417 \mathrm{~cm}^{-1}$ (C-H bending) and $1066 \mathrm{~cm}^{-1}$ due to the stretching of $\mathrm{C}-\mathrm{O} .^{38}$

Fig. 2H-M shows the FTIR spectra of WPI/PPI/GEL and fucoxanthin loaded WPI/PPI/GEL. The infrared spectra of WPI (H), PPI (J) and GEL (L) had very characteristic bands. In the spectra of three kinds native proteins, peaks at around $1532 \mathrm{~cm}^{-1}$ was attributed to $\mathrm{C}=\mathrm{O}$ stretching and $1644-$ $1658 \mathrm{~cm}^{-1}$ attributed to $\mathrm{C}-\mathrm{N}-\mathrm{H}$ in-plane bending and $\mathrm{C}-\mathrm{N}$ stretching, 3292 and $1396 \mathrm{~cm}^{-1}$ attached to $\mathrm{N}-\mathrm{H}$ stretching, and $\mathrm{O}-\mathrm{H}$ bending vibrations of deionized carboxylic acid, respectively, and peaks at $3000-2800$ and $1449 \mathrm{~cm}^{-1}$ correlated to $\mathrm{C}-\mathrm{H}$ stretching and $\mathrm{C}-\mathrm{H}$ bending. ${ }^{39}$ When the FTIR spectra of the six microcapsule powders dried by spray-drying were compared with corresponding wall materials, the appearance of peaks around $1745 \mathrm{~cm}^{-1}(\mathrm{C}=\mathrm{O}$ stretching) was the mainly difference. And all the other absorbance had almost no shifts. The results suggested that the molecular structures of fucoxanthin and wall materials didn't change and there were no new chemical bonds formed. In brief, fucoxanthin was just physically encapsulated inside the microcapsules by hydrogen bonding and van der Waals interactions. ${ }^{40}$

We also investigated proteins' secondary structure and the refolding results are shown in Table 2 . It can be seen from the data that the $\beta$-structures were the most important secondary structure. And there was an expected decrease in $\beta$-sheet, indicating a certain extent of decrease in molecular order. The increase observed in the other bands can be attributed to the conversion of inter-molecular $\beta$-sheet into non-native $\alpha$-helix, $\beta$ turns, and random coil. ${ }^{41}$ 

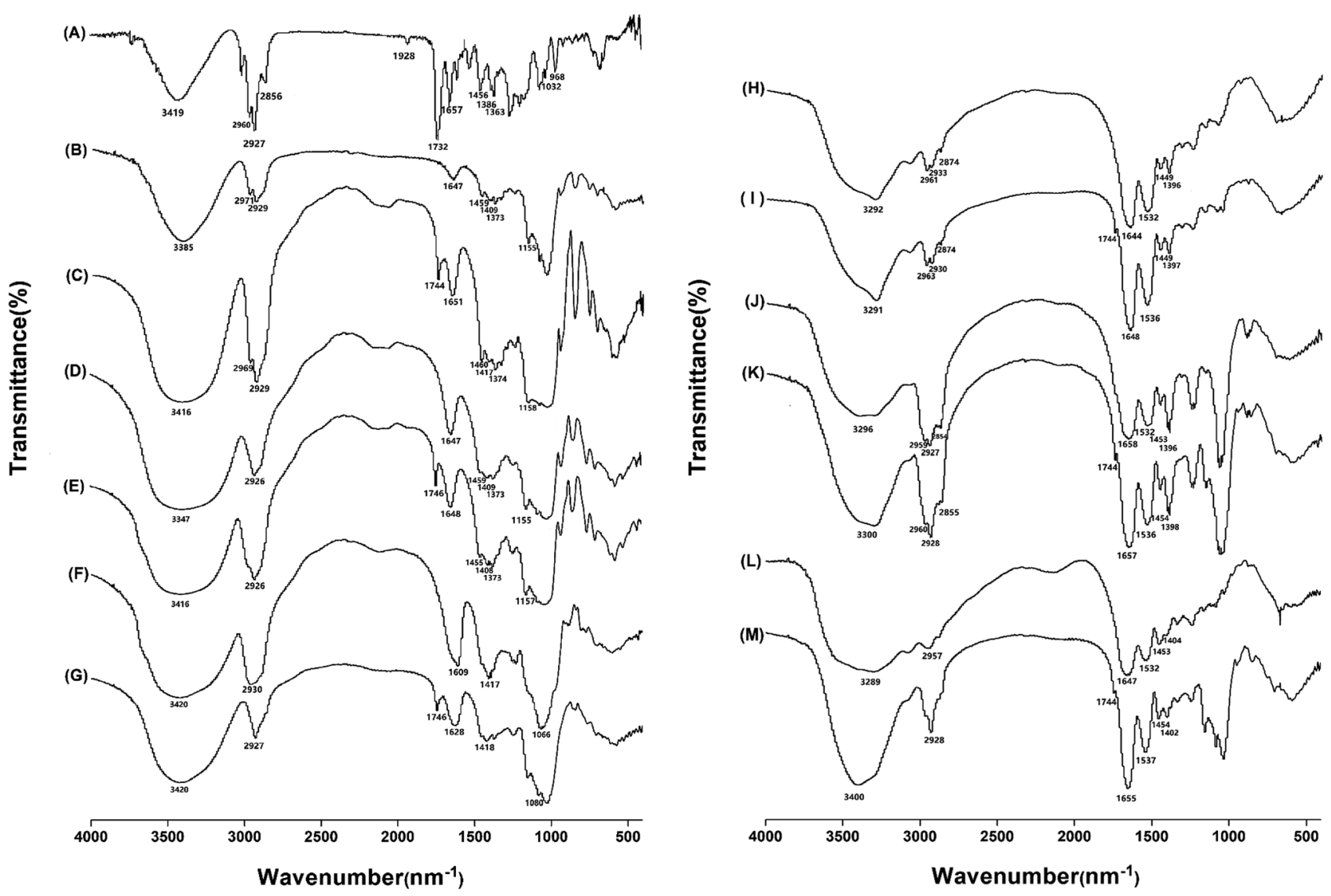

Fig. 2 Fourier transform infrared (FTIR) spectroscopy of fucoxanthin microcapsules. Fucoxanthin (Fx) extracted from Undaria pinnatifida (A), HP$\beta-C D(B), F X-H P-\beta-C D(C), M D(D), F x-M D(E)$. GA (F), Fx-GA (G), WPI (H), Fx-WPI (I), PPI (J), Fx-PPI (K), GEL (L) and Fx-GEL (M). Each FTIR spectrum was analyzed in the wavelength range of $4000-400 \mathrm{~cm}^{-1}$.

\subsection{Heat stability of fucoxanthin loaded microspheres}

Heat stability was evaluated to compare the protective effect of the six selected materials. To study the heat stability of fucoxanthin microcapsules, accelerated thermal experiment was conducted to study the degradations of free fucoxanthin and microencapsulated fucoxanthin under $90^{\circ} \mathrm{C}$.

The degradation of free fucoxanthin reached $2.49 \%$ after heating at $90{ }^{\circ} \mathrm{C}$ for $24 \mathrm{~h}$ (Fig. 3). WPI, GA and MD exerted superior effect on prohibiting fucoxanthin degradation with residual rate of $60.72 \%, 56.03 \%$ and $54.57 \%$ after $24 \mathrm{~h}$, respectively. This was tentatively attributed to the formation of dense layer which might protect wrapped fucoxanthin from damage by heat. Nevertheless, fucoxanthin retention in HP- $\beta$ -
CD was just only $9.32 \%$ after $24 \mathrm{~h}$. According to the previous reports, ${ }^{\mathbf{4 2 - 4 5}}$ we speculated that the cavity structure of HP- $\beta$-CD was modified and enlarged due to the intercalation of fucoxanthin partly, and there was no film formed to protected the content, leading to unsatisfactory protective effect. The conclusion formed that WPI, GA and MD could protect fucoxanthin from heat effectively.

\subsection{Degradation kinetics of fucoxanthin loaded microspheres}

The degradation kinetics of the loaded fucoxanthin encapsulated with different wall materials were studied at $37^{\circ} \mathrm{C}$. The kinetic model of the fucoxanthin degradation profiles was

Table 2 Secondary structure of WPI/PPI/GEL and fucoxanthin loaded microcapsules ${ }^{a}$

\begin{tabular}{|c|c|c|c|c|}
\hline & $\alpha$-helix (\%) & $\beta$-sheet (\%) & $\beta$-turns (\%) & Random coil (\%) \\
\hline WPI & $13.03 \pm 0.31$ & $37.62 \pm 0.31$ & $36.91 \pm 0.16$ & $12.45 \pm 0.09$ \\
\hline Fx-WPI & $14.98 \pm 0.04$ & $32.53 \pm 0.19 *$ & $37.72 \pm 0.14$ & $14.77 \pm 0.02$ \\
\hline PPI & $10.72 \pm 0.59$ & $39.60 \pm 2.21$ & $34.13 \pm 0.21$ & $15.59 \pm 2.56$ \\
\hline Fx-PPI & $13.48 \pm 0.93^{\#}$ & $30.24 \pm 0.04^{\#}$ & $40.02 \pm 0.85^{\#}$ & $16.50 \pm 1.53$ \\
\hline GEL & $13.31 \pm 0.38$ & $35.48 \pm 0.27$ & $37.87 \pm 0.13$ & $13.35 \pm 0.79$ \\
\hline FX-GEL & $14.63 \pm 0.80$ & $31.30 \pm 0.73^{\&}$ & $40.01 \pm 0.45^{\&}$ & $14.06 \pm 0.64$ \\
\hline
\end{tabular}

$a *,{ }^{\#}$, \& represent significant difference compared with WPI, PPI, GEL, respectively $(p<0.05)$. 


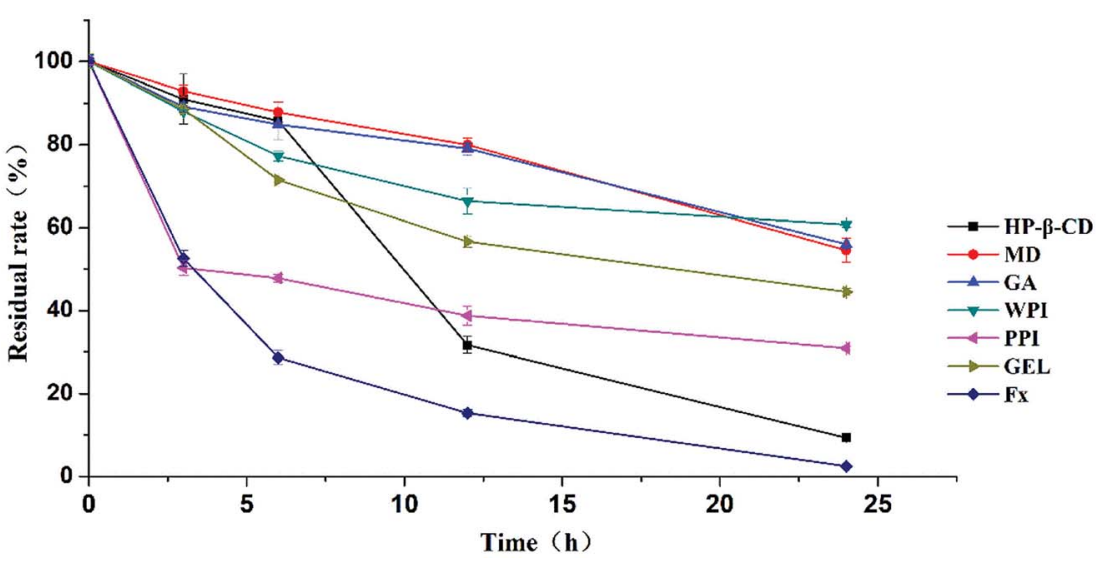

Fig. 3 The heat stability of fucoxanthin microcapsules encapsulated with different wall materials at $90{ }^{\circ} \mathrm{C}$.

established to fit zero-order, first-order or second order kinetics respectively. The best model was chosen based on the highest regression coefficient and the best graphic adjustment. The degradation of fucoxanthin within MD, GA, WPI, and GEL microcapsules fit second-order kinetics equation, within HP- $\beta$ CD and PPI fit zero-order kinetics equation (Table 3, Fig. 4). And the coefficients of $x$ could response the degradation rate. The half-life was calculated by formula (5) and (7) for zero-order degradation kinetics and second-order degradation kinetics, and the results were $26.3 \mathrm{~d}, 92.6 \mathrm{~d}, 99.8 \mathrm{~d}, 50.9 \mathrm{~d}, 69.0 \mathrm{~d}$, and 69.3 d, respectively. Fucoxanthin in all the six samples showed different degradation rates, indicating the type of wall material modulate the degradation of the carotenoid pigment.

Although HP- $\beta$-CD is an excellent carrier for small molecular substances for its special structure, the half-life of fucoxanthin was only $26.3 \mathrm{~d}$. We assume that fucoxanthin partly inserted into the cavity of HP- $\beta$-CD and formed a supramolecular inclusion complex, providing moderate protection to fucoxanthin from degradation by heat and oxygen. However, the long conjugated polyene chains of fucoxanthin were still exposed, which made it protected not quite well. The degradation rates of fucoxanthin in MD and GA were much lower than those in the other wall materials, indicating that they were better in protecting fucoxanthin than the rest four. According to literatures, MD could form dense layer around the encapsulated compounds to protect them against oxygen, moisture and heat during storage. The microcapsule composed of GA was more stable than others, which was probable due to the structure of GA. GA is a highly branched heteropolymer consisting of arabinogalactans, glycoproteins, and arabinogalactan proteins. In this mix, the carbohydrates provide structure through glass formation, and the proteins endow GA the ability of film-forming and emulsifying. ${ }^{46}$ Consequently, of all the wall materials, GA presented the best protection of fucoxanthin with a half-life of $99.8 \mathrm{~d}$. These results indicated that GA and MD had a better protective effect on fucoxanthin.

\subsection{Stability and bioaccessibility of fucoxanthin in vitro digestion}

Fucoxanthin can be easily degraded in acidic environment owing to the electrophilicity of its polyunsaturated bonds and it is exposed to gastric acid during the digestion process in the gastric stage. The other issue that matters is the bioaccessibility, for it could reflect the absorption of fucoxanthin into blood circulation. The main absorption sites of fucoxanthin are located in small intestine. The six microcapsules were tested in simulated digestion process to evaluate their digestive stability and bioaccessibility. According to Koo et al.'s method, ${ }^{31}$ the whole collected digestion solutions from gastric stage were analyzed to evaluate the digestive stability of fucoxanthin in the simulated gastric juice, and the micelle obtained by centrifugation of the solution collected in the simulated small intestine stages was used to evaluate the bioaccessibility of fucoxanthin correspondingly.

Table 3 Degradation parameters of fucoxanthin degradation in microcapsule samples

\begin{tabular}{|c|c|c|c|c|c|c|}
\hline HP- $\beta-C D$ & $-3.45 \pm 0.19$ & 0.9821 & - & - & $1.52 \pm 0.82$ & 0.3260 \\
\hline $\mathrm{MD}$ & $-1.13 \pm 0.21$ & 0.8241 & - & - & $0.61 \pm 0.08$ & 0.9217 \\
\hline GA & $-1.52 \pm 0.13$ & 0.9558 & - & - & $0.76 \pm 0.03$ & 0.9913 \\
\hline GEL & $-0.93 \pm 0.14$ & 0.8965 & $-9.92 \pm 1.21$ & 0.9292 & $1.26 \pm 0.09$ & 0.9983 \\
\hline
\end{tabular}




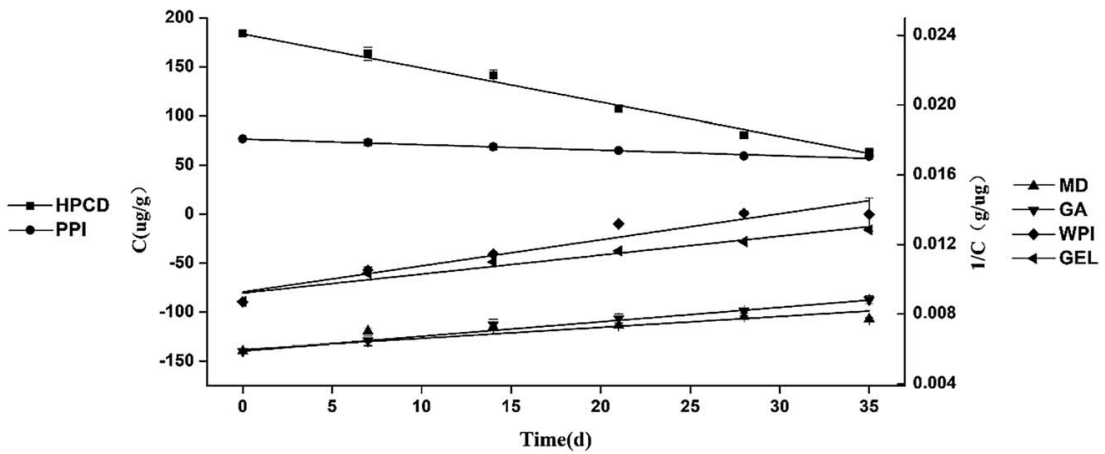

Fig. 4 Fucoxanthin degradation kinetics of microcapsules based on different vehicles: HP- $\beta-C D, M D, G A, W P I, P P I$ and GEL at the temperature of $37^{\circ} \mathrm{C}$.

As shown in Fig. 5, fucoxanthin in all the six microcapsules degraded with varying degrees through the simulated gastric tract digestion process, the retention rate of fucoxanthin was around $68.82-89.38 \%$, while fucoxanthin free decreased to
$61.19 \%$. Among them, fucoxanthin remained over $80 \%$ embedded in MD, GA and WPI. This result indicated that fucoxanthin in the six kinds of microcapsules were more stable in the gastric digestion environment. In the small intestine
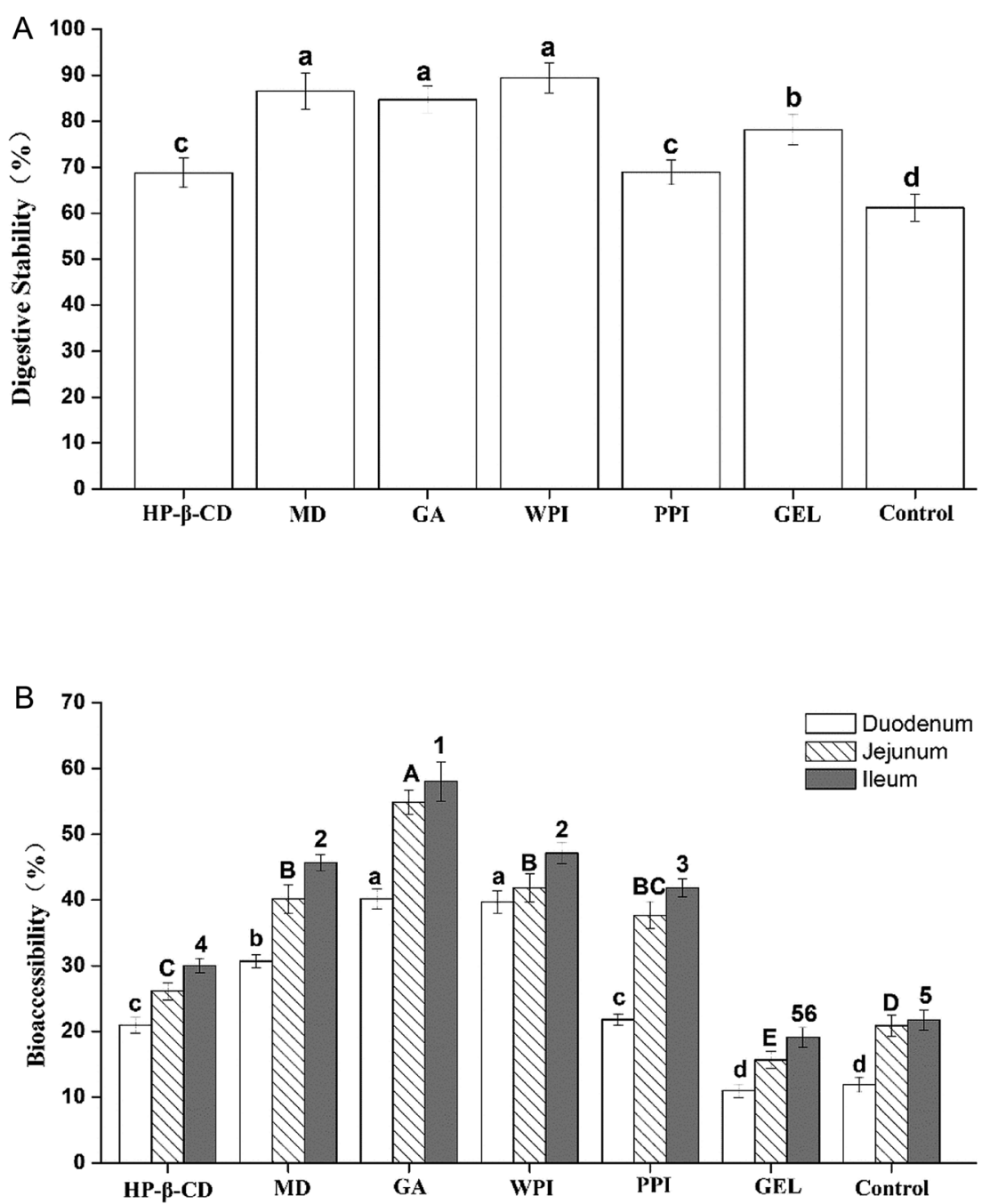

Fig. 5 (A) Digestive stability (\%) of fucoxanthin in microcapsules after simulated gastric tract digestion in vitro. (B) Bioaccessibility (\%) of fucoxanthin in microcapsules after three intestinal tracts digestion in vitro. 
stages of the simulated digestion in vitro, the fucoxanthin released to the micelle phases of Fx-HP- $\beta-\mathrm{CD}$, Fx-MD, Fx-GA, FxWPI and Fx-PPI was in the range of $21-30 \%, 31-46 \%, 40-58 \%$, $40-47 \%$ and $22-42 \%$, respectively, which was higher than that of fucoxanthin-free (10-21\%) and Fx-GEL (11-20\%). Considering the retention rate in the previous phase, MD, GA, WPI were more suitable for protecting fucoxanthin from degradation in the digestive process and fucoxanthin can be released more easily.

\section{Conclusion}

In this paper, fucoxanthin microcapsules were prepared using six biopolymers as carriers by spray drying. The ones with carbohydrate were sphere with little shrinking and had more concave on the surface of microcapsules made by proteins. The mean particle diameter of the protein based microparticles was a bit bigger than that of the carbohydrate based microparticles. All the microcapsules had low moisture content and $a_{\mathrm{w}}$, and good encapsulation efficiency.

FTIR analysis showed that it was a physical encapsulation process and no new chemical bonds formed between fucoxanthin and biopolymers. WPI, GA and MD showed effective protection on fucoxanthin against heating at high temperature. And the encapsulated fucoxanthin degradation in GA and MD followed the second-order reaction and were more suitable for protecting fucoxanthin. In the digestion process in vitro, it showed that MD, GA and WPI had a better ability to prevent the degradation and fucoxanthin can be released more easily in the intestinal tract. This study provided useful information for the microencapsulation of fucoxanthin in further studies and industrial application.

\section{Conflicts of interest}

There are no conflicts of interest to declare.

\section{Acknowledgements}

This work was supported by the Independent Innovation Major Project of Huangdao District Qingdao City (Grant No. 2014-311); and the Open Foundation of the State Key Laboratory of Bioactive Seaweed Substances (Grant No. SKL-BASS1704).

\section{References}

1 H. Maeda, M. Hosokawa, T. Sashima, K. Funayama and K. Miyashita, Fucoxanthin from edible seaweed, Undaria pinnatifida, shows antiobesity effect through UCP1 expression in white adipose tissues, Biochem. Biophys. Res. Commun., 2005, 332(2), 392-397.

2 K. Miyashita and M. Hosokawa, Fucoxanthin in the management of obesity and its related disorders, J. Funct. Foods, 2017, 36, 195-202.

3 H. Maeda, M. Hosokawa, T. Sashima, K. MurakamiFunayama and K. Miyashita, Anti-obesity and anti-diabetic effects of fucoxanthin on diet-induced obesity conditions in a murine model, Mol. Med. Rep., 2009, 2(6), 897-902.

4 A. Fung, N. Hamid and J. Lu, Fucoxanthin content and antioxidant properties of Undaria pinnatifida, Food Chem., 2013, 136(2), 1055-1062.

5 S. J. Heo, W. J. Yoon, K. N. Kim, G. N. Ahn, S. M. Kang, D. H. Kang, A. Affan, C. Oh, W. K. Jung and Y. J. Jeon, Evaluation of anti-inflammatory effect of fucoxanthin isolated from brown algae in lipopolysaccharide-stimulated RAW 264.7 macrophages, Food Chem. Toxicol., 2010, 48(8), 2045-2051.

6 R. Pangestuti, T. S. Vo, D. H. Ngo and S. K. Kim, Fucoxanthin ameliorates inflammation and oxidative responses in microglia, J. Agric. Food Chem., 2013, 61(16), 3876-3883.

7 E. Kotake-Nara, A. Asai and A. Nagao, Neoxanthin and fucoxanthin induce apoptosis in PC-3 human prostate cancer cells, Cancer Lett., 2005, 220(1), 75-84.

8 S. R. Kumar, M. Hosokawa and K. Miyashita, Fucoxanthin: A marine carotenoid exerting anti-cancer effects by affecting multiple mechanisms, Mar. Drugs, 2013, 11(12), 5130-5147.

9 E. Pinto, T. Sigaud-kutner, M. A. Leitao, O. K. Okamoto, D. Morse and P. Colepicolo, Heavy metal-induced oxidative stress in algae1, J. Phycol., 2003, 39(6), 1008-1018.

10 H. SiewLing, C. PooiYi, W. KwanKit and W. ChingLee, Stability studies of fucoxanthin from Sargassum binderi, Aust. J. Basic Appl. Sci., 2010, 4(10), 4580-4584.

11 D. Zhao, S. M. Kim, C. H. Pan and D. Chung, Effects of heating, aerial exposure and illumination on stability of fucoxanthin in canola oil, Food Chem., 2014, 145, 505-513.

12 T. Hashimoto, Y. Ozaki, M. Mizuno, M. Yoshida, Y. Nishitani, T. Azuma, A. Komoto, T. Maoka, Y. Tanino and K. Kanazawa, Pharmacokinetics of fucoxanthinol in human plasma after the oral administration of kombu extract, Br. J. Nutr., 2012, 107(11), 1566-1569.

13 E. G. Donhowe and F. Kong, Beta-carotene: digestion, microencapsulation, and in vitro bioavailability, Food Bioprocess Technol., 2014, 7(2), 338-354.

14 B. Shu, W. Yu, Y. Zhao and X. Liu, Study on microencapsulation of lycopene by spray-drying, J. Food Eng., 2006, 76(4), 664-669.

15 S. M. Jafari, E. Assadpoor, Y. He and B. Bhandari, Encapsulation efficiency of food flavours and oils during spray drying, Drying Technol., 2008, 26(7), 816-835.

16 N. Mahfoudhi and S. Hamdi, Kinetic degradation and storage stability of $\beta$-carotene encapsulated by spray drying using almond gum and gum arabic as wall materials, $J$. Polym. Eng., 2014, 34(8), 683-693.

17 M. A. Montenegro, I. L. Nunes, A. Z. Mercadante and C. D. Borsarelli, Photoprotection of Vitamins in Skimmed Milk by an Aqueous Soluble Lycopene-Gum Arabic Microcapsule, J. Agric. Food Chem., 2007, 55(2), 323-329.

18 N. Anarjan, C. P. Tan, I. A. Nehdi and T. C. Ling, Colloidal astaxanthin: Preparation, characterisation and bioavailability evaluation, Food Chem., 2012, 135(3), 13031309.

19 C. Bustos-Garza, J. Yáñez-Fernández and B. E. BarragánHuerta, Thermal and $\mathrm{pH}$ stability of spray-dried 
encapsulated astaxanthin oleoresin from Haematococcus pluvialis using several encapsulation wall materials, Food Res. Int., 2013, 54(1), 641-649.

20 C. Chranioti, A. Nikoloudaki and C. Tzia, Saffron and beetroot extracts encapsulated in maltodextrin, gum Arabic, modified starch and chitosan: Incorporation in a chewing gum system, Carbohydr. Polym., 2015, 127, 252263.

21 F. Gandía-Herrero, M. Jiménez-Atiénzar, J. Cabanes, F. García-Carmona and J. Escribano, Stabilization of the bioactive pigment of Opuntia fruits through maltodextrin encapsulation, J. Agric. Food Chem., 2010, 58(19), 1064610652.

22 S. M. T. Gharibzahedi, S. H. Razavi and M. Mousavi, Characterizing the natural canthaxanthin/2-hydroxypropyl$\beta$-cyclodextrin inclusion complex, Carbohydr. Polym., 2014, 101, 1147-1153.

23 C. Yuan, L. Du, Z. Jin and X. Xu, Storage stability and antioxidant activity of complex of astaxanthin with hydroxypropyl- $\beta$-cyclodextrin, Carbohydr. Polym., 2013, 91(1), 385-389.

24 M. A. Stout, C. W. Park and M. A. Drake, The effect of bleaching agents on the degradation of vitamins and carotenoids in spray-dried whey protein concentrate, $J$. Dairy Sci., 2017, 100(10), 7922-7932.

25 N. Anarjan, I. A. Nehdi, H. M. Sbihi, S. I. Al-Resayes, H. J. Malmiri and C. P. Tan, Preparation of astaxanthin nanodispersions using gelatin-based stabilizer systems, Molecules, 2014, 19(9), 14257-14265.

26 S. Uzun, H. Kim, C. Leal and G. W. Padua, Ethanol-induced whey protein gels as carriers for lutein droplets, Food Hydrocolloids, 2016, 61, 426-432.

27 J. Yi, Y. Fan, W. Yokoyama, Y. Zhang and L. Zhao, Characterization of milk proteins-lutein complexes and the impact on lutein chemical stability, Food Chem., 2016, 200, 91-97.

28 J. Zhu, X. Sun, X. Chen, S. Wang and D. Wang, Chemical cleavage of fucoxanthin from Undaria pinnatifida and formation of apo-fucoxanthinones and apo-fucoxanthinals identified using LC-DAD-APCI-MS/MS, Food Chem., 2016, 211, 365-373.

29 S. M. Rivera and R. Canela-Garayoa, Analytical tools for the analysis of carotenoids in diverse materials, J. Chromatogr. A, 2012, 1224, 1-10.

30 B. Wang, B. Adhikari and C. J. Barrow, Optimisation of the microencapsulation of tuna oil in gelatin-sodium hexametaphosphate using complex coacervation, Food Chem., 2014, 158, 358-365.

31 S. Y. Koo, I. K. Mok, C. H. Pan and S. M. Kim, Preparation of Fucoxanthin-Loaded Nanoparticles Composed of Casein and Chitosan with Improved Fucoxanthin Bioavailability, $J$. Agric. Food Chem., 2016, 64(49), 9428-9435.

32 E. G. Donhowe, F. P. Flores, W. L. Kerr, L. Wicker and F. Kong, Characterization and in vitro bioavailability of $\beta$ carotene: Effects of microencapsulation method and food matrix, LWT-Food Sci. Technol., 2014, 57(1), 42-48.
33 T. A. Comunian, E. S. Monterrey-Quintero, M. Thomazini, J. C. Balieiro, P. Piccone, P. Pittia and C. S. Favaro-Trindade, Assessment of production efficiency, physicochemical properties and storage stability of spray-dried chlorophyllide, a natural food colourant, using gum Arabic, maltodextrin and soy protein isolate-based carrier systems, Int. J. Food Sci. Technol., 2011, 46(6), 12591265.

34 A. Y. Guadarrama-Lezama, L. Dorantes-Alvarez, M. E. Jaramillo-Flores, C. Pérez-Alonso, K. Niranjan, G. F. Gutiérrez-López and L. Alamilla-Beltrán, Preparation and characterization of non-aqueous extracts from chilli (Capsicum annuum L.) and their microencapsulates obtained by spray-drying, J. Food Eng., 2012, 112(1-2), 29-37.

35 V. Castel, A. C. Rubiolo and C. R. Carrara, Brea gum as wall material in the microencapsulation of corn oil by spray drying: Effect of inulin addition, Food Res. Int., 2018, 103, 76-83.

36 H. Ravi and V. Baskaran, Biodegradable chitosan-glycolipid hybrid nanogels: A novel approach to encapsulate fucoxanthin for improved stability and bioavailability, Food Hydrocolloids, 2015, 43, 717-725.

37 X. Wang, H. Li, F. Wang, G. Xia, H. Liu, X. Cheng, M. Kong, Y. Liu, C. Feng, X. Chen and Y. Wang, Isolation of fucoxanthin from Sargassum thunbergii and preparation of microcapsules based on palm stearin solid lipid core, Front. Mater. Sci., 2017, 11(1), 66-74.

38 J. S. García-Saldaña, O. N. Campas-Baypoli, J. LópezCervantes, D. I. Sánchez-Machado, E. U. Cantú-Soto and R. Rodríguez-Ramírez, Microencapsulation of sulforaphane from broccoli seed extracts by gelatin/gum arabic and gelatin/pectin complexes, Food Chem., 2016, 201, 94-100.

39 L. Bagheri, A. Madadlou, M. Yarmand and M. E. Mousavi, Nanoencapsulation of date palm pit extract in whey protein particles generated via desolvation method, Food Res. Int., 2013, 51(2), 866-871.

40 M. Ahmadi, A. Madadlou and A. A. Sabouri, Isolation of micro-and nano-crystalline cellulose particles and fabrication of crystalline particles-loaded whey protein cold-set gel, Food Chem., 2015, 174, 97-103.

41 D. Oboroceanu, L. Wang, A. Kroes-Nijboer, A. Brodkorb, P. Venema, E. Magner and M. A. Auty, The effect of high pressure microfluidization on the structure and length distribution of whey protein fibrils, Int. Dairy J., 2011, 21(10), 823-830.

42 J. Chao, D. Meng, J. Li, H. Xu and S. Huang, Preparation and study on the novel solid inclusion complex of ciprofloxacin with HP- $\beta$-cyclodextrin, Spectrochim. Acta, Part A, 2004, 60(3), 729-734.

43 Y. Deng, Y. Pang, Y. Guo, Y. Ren, F. Wang, X. Liao and B. Yang, Host-guest inclusion systems of daidzein with 2hydroxypropyl- $\beta$-cyclodextrin (HP- $\beta$-CD) and sulfobutyl ether- $\beta$-cyclodextrin (SBE- $\beta$-CD): Preparation, binding behaviors and water solubility, J. Mol. Struct., 2016, 1118, 307-315.

44 J. Li, M. Zhang, J. Chao and S. Shuang, Preparation and characterization of the inclusion complex of Baicalin (BG) 
with $\beta$-CD and HP- $\beta$-CD in solution: an antioxidant ability study, Spectrochim. Acta, Part A, 2009, 73(4), 752-756.

45 T. A. Nguyen, B. Liu, J. Zhao, D. S. Thomas and J. M. Hook, An investigation into the supramolecular structure, solubility, stability and antioxidant activity of rutin/ cyclodextrin inclusion complex, Food Chem., 2013, 136(1), 186-192.

46 R. C. Randall, G. O. Phillips and P. A. Williams, Fractionation and characterization of gum from Acacia senegal, Food Hydrocolloids, 1989, 3(1), 65-75. 